\title{
Novel Mutations in NPCI are Associated with Pelizaeus-Merzbacher-Like Disease: A Case Report
}

This article was published in the following Dove Press journal: International Journal of General Medicine

\author{
Hongling $\mathrm{Fu}^{\mathrm{l}, 2}$ \\ Qiu Wang ${ }^{2,3}$ \\ Hanmin Liu ${ }^{1,2}$ \\ 'Department of Pediatrics, West China \\ Second University Hospital, Sichuan \\ University, Chengdu, Sichuan, People's \\ Republic of China; ${ }^{2}$ Key Laboratory of \\ Birth Defect and Related Diseases of \\ Women and Children (Sichuan \\ University), Ministry of Education, West \\ China Second University Hospital, \\ Sichuan University, Chengdu, Sichuan, \\ People's Republic of China; ${ }^{3}$ Department \\ of Rehabilitation Medicine, West China \\ Second University Hospital, Sichuan \\ University, Chengdu, Sichuan, People's \\ Republic of China
}

Correspondence: Hanmin Liu Department of Pediatrics, West China Second University Hospital, Sichuan University, No. 20, 3rd Section, Ren Min Nan Lu, Chengdu, 61004I, People's Republic of China

Email liuhm@scu.edu.cn

\begin{abstract}
Pelizaeus-Merzbacher-like disease (PMLD) is an autosomal recessive hypomyelinating leukodystrophy with clinical symptoms and imaging manifestations similar to those of Pelizaeus-Merzbacher disease (PMD), an X-linked recessive hypomyelinating leukodystrophy. Typical manifestations of PMLD are nystagmus, dysmyotonia, ataxia, progressive motor dysfunction, and diffuse leukodystrophy on magnetic resonance imaging (MRI). This report identified novel mutations in NCP1 causing PMLD. A 7-monthold male patient was referred to our hospital because he could not lift his head until that time. He had symptoms including congenital nystagmus, hypotonia, and developmental delay. According to the MRI scan, there were signs of leukodystrophy. According to the clinical manifestations and the results of whole-exome sequencing (compound heterozygote mutations in NPC1 (p. G911S, c2731G $>$ A and p. D128H, c382G $>C$ )), the diagnosis of PMLD was considered, and his parents were determined to be carriers of mutant genes. He began rehabilitation training at the age of 1 year old. After 5 years of training, he was still experiencing global developmental delay, equivalent to the developmental level of a nine-month-old child. PMLD is a disease that seriously affects the quality of life of children and can result from mutations in different genes. In this report, we expand the gene spectrum of PMLD and suggest early genetic counselling for suspected patients and their patients.
\end{abstract}

Keywords: PMLD, hypomyelinating leukodystrophy, NPC, compound heterozygote mutations

\section{Introduction}

Pelizaeus-Merzbacher disease (PMD; MIM\#312080) is an X-linked recessive hypomyelinating leukodystrophy-1 (HLD1) that presents in infancy with hypotonia, nystagmus, cognitive impairment, ataxia, severe spasticity, and respiratory distress. ${ }^{1}$ The disorder is caused by mutations or rearrangements in the PLP1 gene, located on chromosome $\mathrm{Xq} 22$ and encoding myelin proteolipid protein a major and essential component of the CNS. ${ }^{1,2}$ The prevalence of PMD is estimated to be 1: 300,000-1: 500,000 among American males. PelizaeusMerzbacher-like disease (PMLD; MIM\#608804), with clinical manifestations associated with hypomyelination, is an autosomal recessive disorder. To date, PMLD has been termed a disorder with a clinically similar phenotype associated with hypomyelination but not linked to PLP1. ${ }^{3}$ Therefore, PMLD can show genetic heterogeneity depending on different mutant genes. 
In our case, we found mutations in NPC1 (p. G911S, and 2). NPC1, encoding a transmembrane protein of the c2731G $>$ A and p. D128H, c382G $>$ C) in a patient with acidic compartment, has been associated with Niemannsimilar clinical characteristics of PMLD (Figures 1 Pick type C1 (NPC1) disease. Niemann-Pick disease type

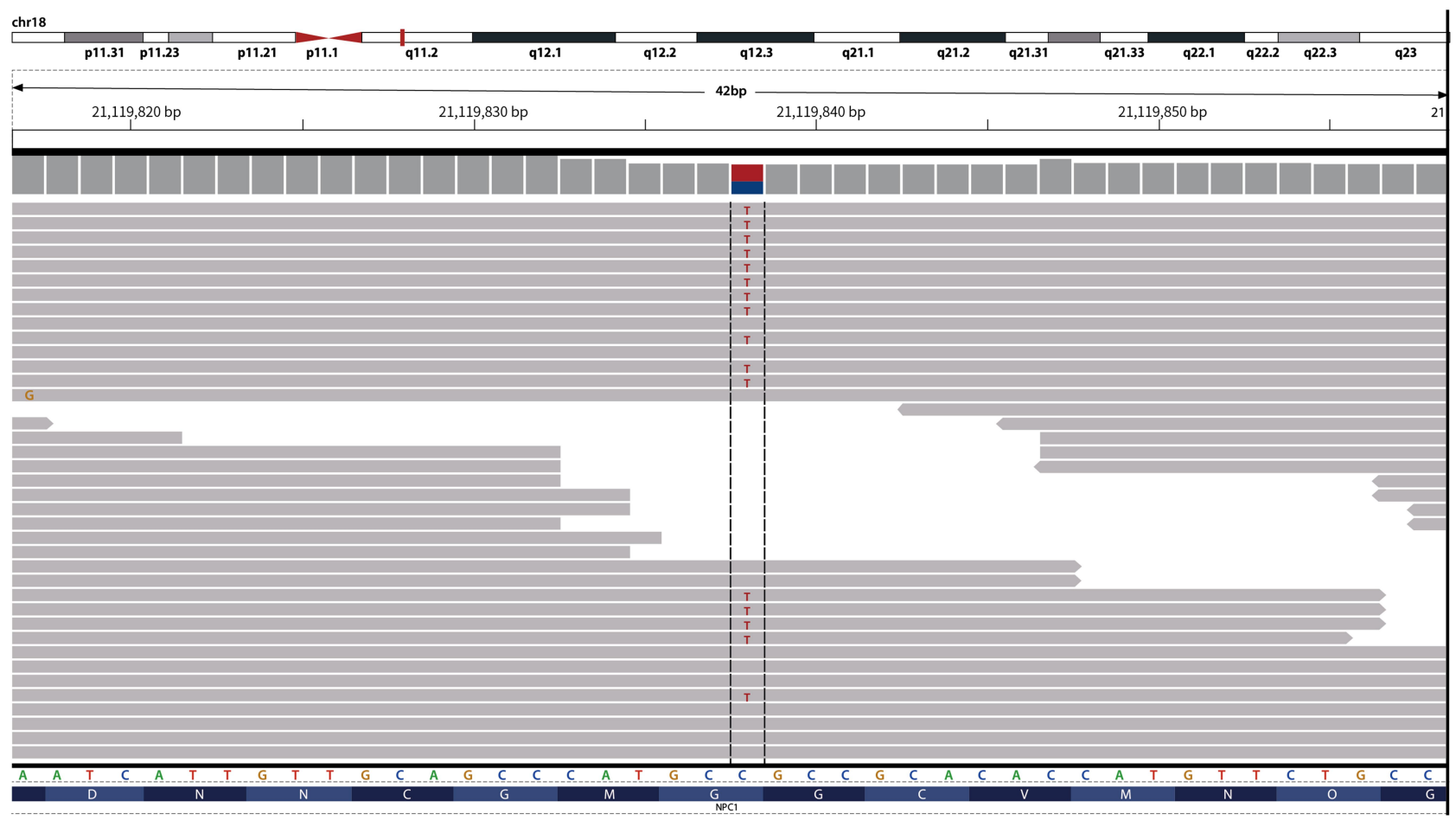

Figure I c273IG>A in exon 18 (chrl 8: 2I I I 9839) caused point mutation which resulted in serine replaced by glycine (p.G9IIS).

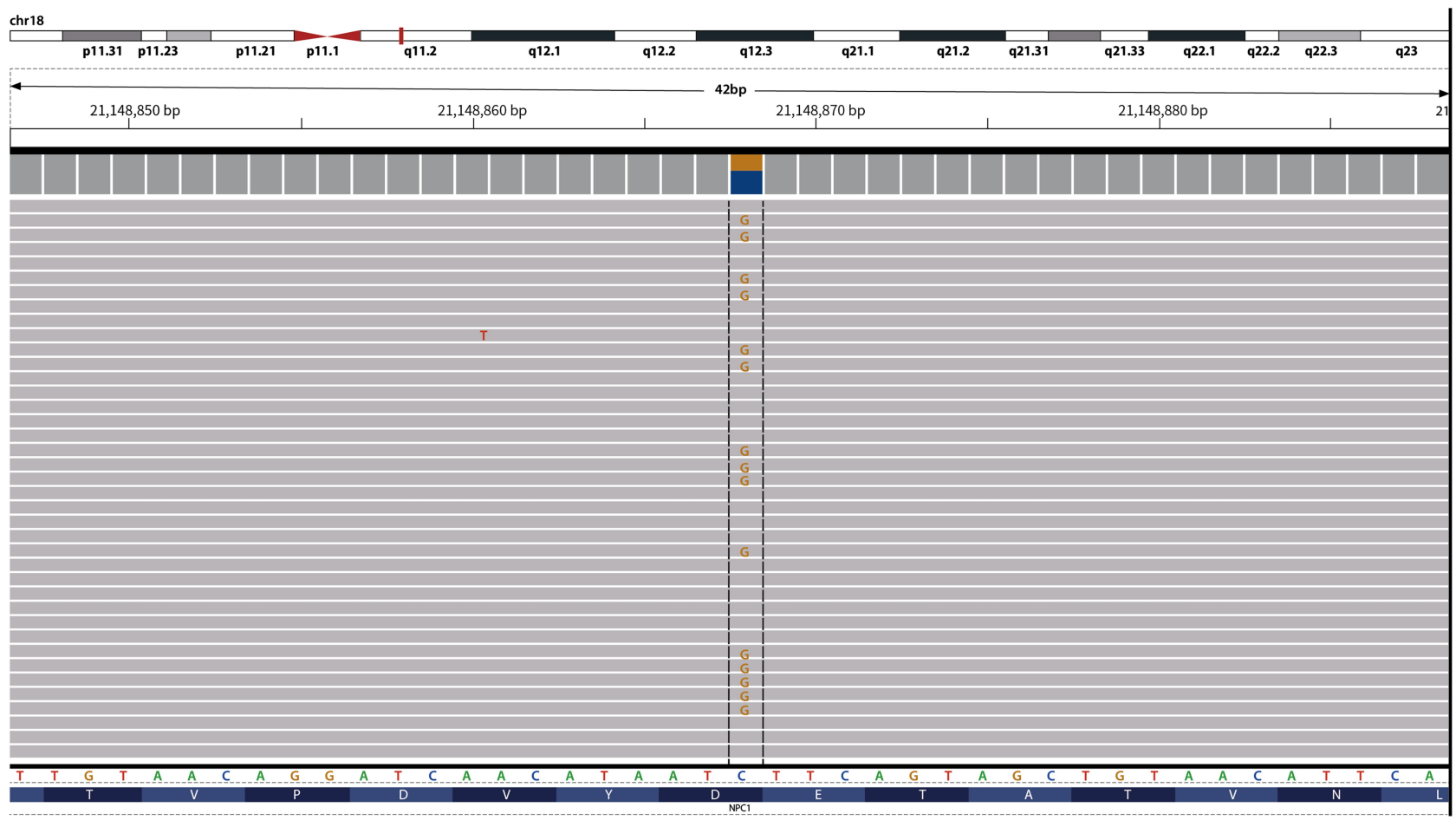

Figure 2 c382G $>C$ in exon 4 (chcr I8: 2/ |48868) caused point mutation which resulted in histidine replaced by aspartic acid (p.DI28H). 
$\mathrm{C}$ (NPC) is an autosomal recessive lysosomal lipid storage disease caused by mutations of the NPC1 gene. ${ }^{3,4}$ It mainly affects the internal organs and nervous system. Persistent neonatal cholestatic jaundice, hepatosplenomegaly, dystaxia, dysphagia, dysarthria and dysgnosia are characteristic manifestations of the disorder. ${ }^{5}$ Patients vary in age from newborn to adult, though the disease manifests more often in childhood. Moreover, due to the age of onset, the range of lesions involved is not consistent, and the clinical manifestations have obvious heterogeneity. ${ }^{6}$ Generally, the earlier the age of onset, the more serious the state of the disease. Although the first neurological symptoms are different and there is obvious clinical heterogeneity, prolonged neonatal cholestasis, hepatosplenomegaly or isolated cataplexy, splenomegaly, and vertical supranuclear gaze palsy are typical symptoms of the disease. The dysfunction of NPC genes, such as NPC1 and NPC2, leads to impaired cholesterol trafficking and thus causes intracellular accumulation of cholesterol and glycolipids in late endosomes and lysosomes. ${ }^{7}$ Dysfunctional cellular autophagy, defective lysosomal calcium homeostasis and oxidative stress play important roles in the pathophysiological processes of the disease.

In our case, we noticed a male child who had a typical PMLD phenotype; on an MRI scan, there were signs of leukodystrophy. We performed whole-exome sequencing (WES) in this patient and found mutations in NPC1 rather than variants in genes attributed to PMD or PMLD, such as PLP1 or GJC2. Moreover, no mutations related to leukodystrophy were identified. Therefore, our findings expand the genetic spectrum of PMLD.

\section{Ethical Approval}

The patients' parents (as his legal guardian) provided signed informed consent for genetic analysis and publication. Our study was approved by the Ethics Committee of West China Second Hospital of Sichuan University, Chengdu, China.

\section{Case Presentation}

The patient was G2P1 (the mother had a history of induced abortion), born by vaginal delivery. His father was 27 years old and his mother was 26 years old when he was born. During pregnancy, the mother had threatened abortion before 12 weeks, and she took Chinese medicine as antiparturition therapy. The delivery went smoothly. The patient's gestational age was $39+2 \mathrm{~W}$, and his birth weight was $3350 \mathrm{~g}$. Moreover, the patients denied a history of neonatal asphyxia, jaundice, convulsion, and related symptoms during the patient's neonatal period, but when mouth feeding, the patient often choked on milk and spit milk and experienced overflow. The patient was first referred to neurology when he was 7 months old because he could not lift his head until that time. Our doctor performed a physical examination, which revealed that he could not hold his head to maintain a neutral position or sit by himself. He also had horizontal nystagmus and hypotonia. MRI of the patient's brain at 7 months of age showed abnormal symmetry signals of the bilateral pallidus, diffuse symmetry abnormal white matter signals of the bilateral cerebral hemispheres and cerebellum, and delayed development of the brain myelin sheath, suggesting the possibility of hereditary metabolic encephalopathy (Figures 3 and 4). Urine metabolism testing showed that there were increases in Leu/Ala and $\mathrm{C} 4 / \mathrm{C} 3$ and decreases in Thr, $\mathrm{C} 0 / \mathrm{C} 3$ and $\mathrm{C} 3 / \mathrm{C} 2$. No specific fatty acid metabolism abnormalities were suspected. The peripheral nerve function of the patient's lower limbs was normal. DNA microarray examination showed no chromosomal abnormalities at 1440 genetic sites on 46 chromosomes. Wholeexome sequencing (WES) showed no mutations in genes

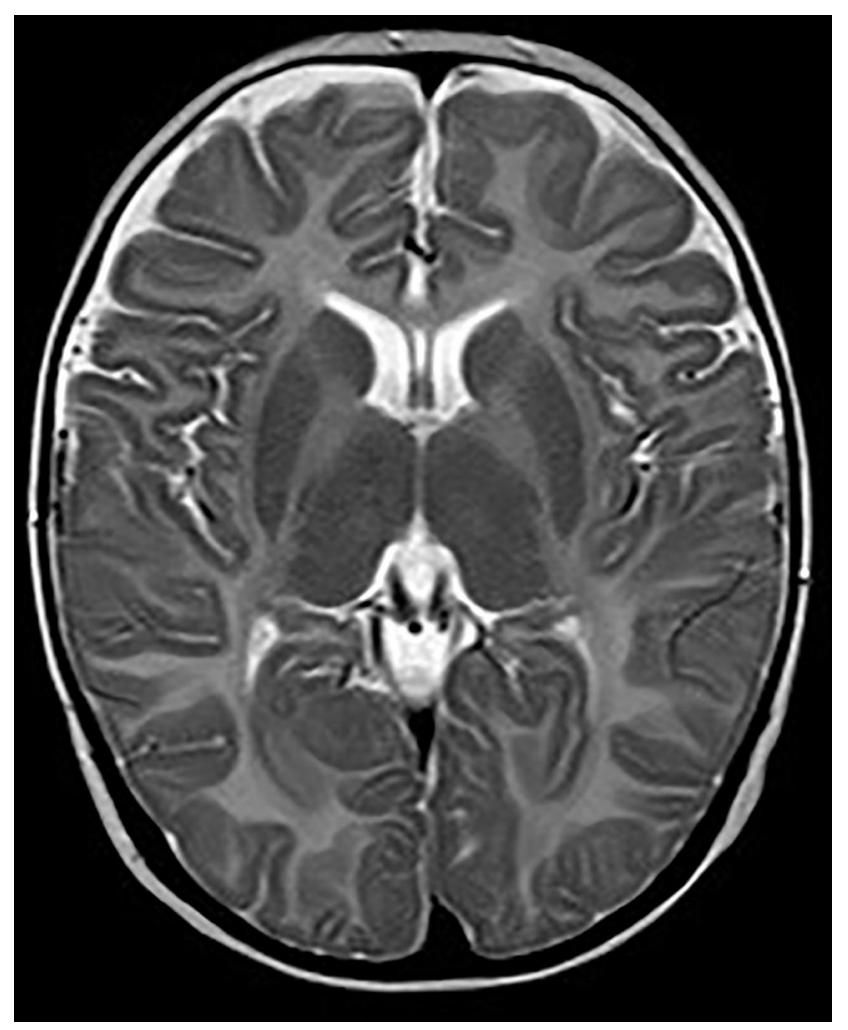

Figure 3 Axial MRI images at age of 7 months showing abnormal symmetry signals of bilateral pallidus. 


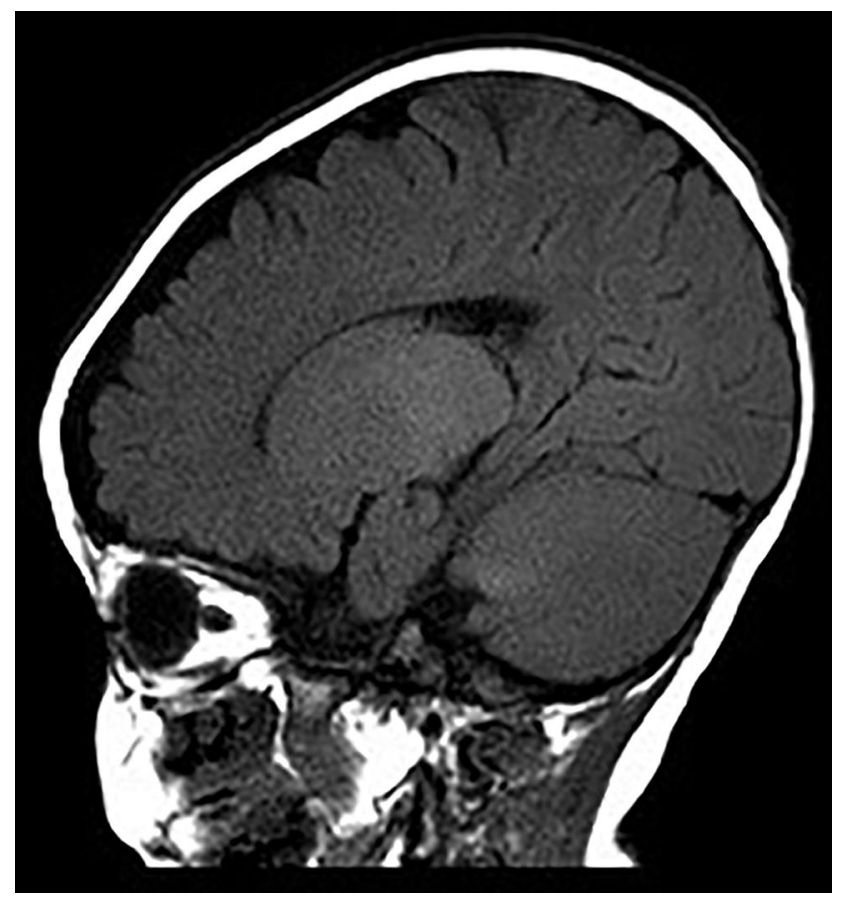

Figure 4 Sagittal MRI images at age of 7 months showing diffuse symmetry abnormal white matter signals of bilateral cerebral hemispheres and cerebellum, and delayed development of brain myelin sheath.

related to PMD and PMLD (such as PLP1 and GJC2) or variants in genes related to leukodystrophy, such as HSPD1. The MRI scans were repeated when he was 9 months and 18 months old. The images at the age of 9 months displayed diffusely and symmetrically abnormal signals in the white matter in the bilateral cerebral hemispheres, cerebellar abnormality, and bilateral pallidus, suggesting myelin formation disorder (Figures 5 and 6).

The patient began rehabilitation training at the age of 1 year old. He is 6 years old now and has been treated for 5 years. Rehabilitation assessment suggests that his motor and mental scales are lagging significantly behind. To date, he can only say 1-2 meaningful words, such as consciously call mom and dad, but the pronunciation is not very clear. He can understand simple instruction; for example, he can roll over with difficulty when he hears the instruction "roll over". Sitting alone remains impossible, and eating is completely dependent on help. The symptom of nystagmus has improved. Other symptoms include hypermyotonia of both lower extremities, talipes varus and inflexion.

As mentioned above, the patient had early-onset nystagmus, dysmyotonia, motor and mental retardation

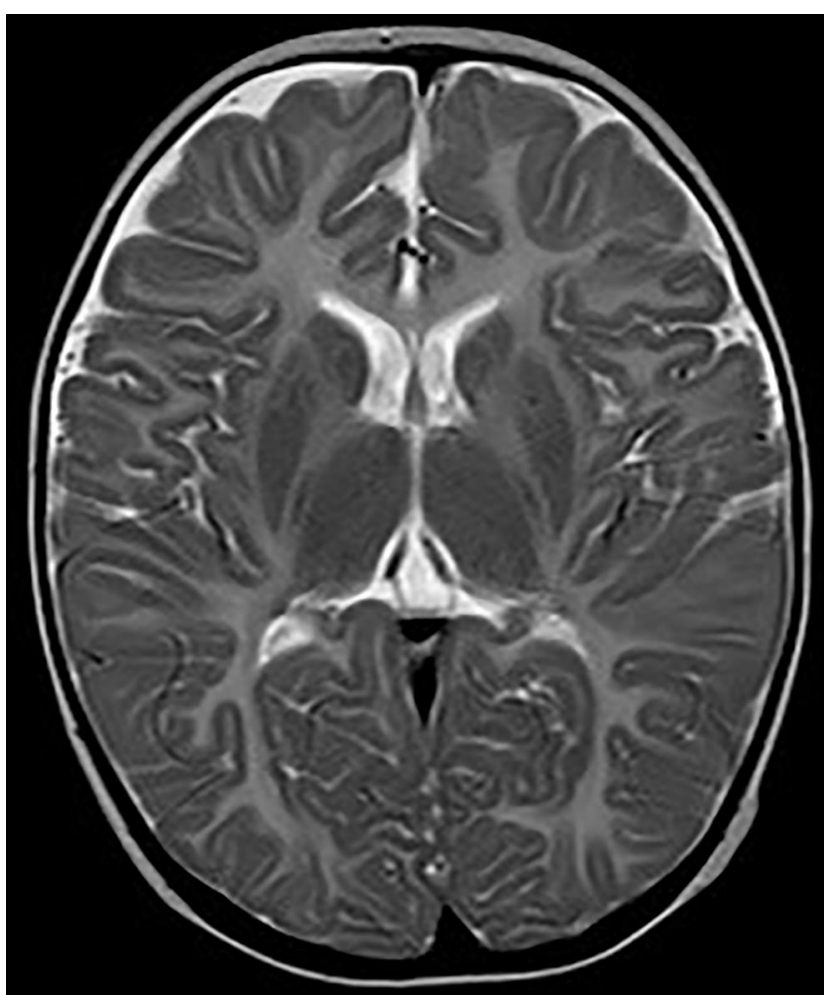

Figure 5 Axial MRI images at age of 9 months showing displayed diffusely and symmetrically abnormal signal in bilateral pallidus.

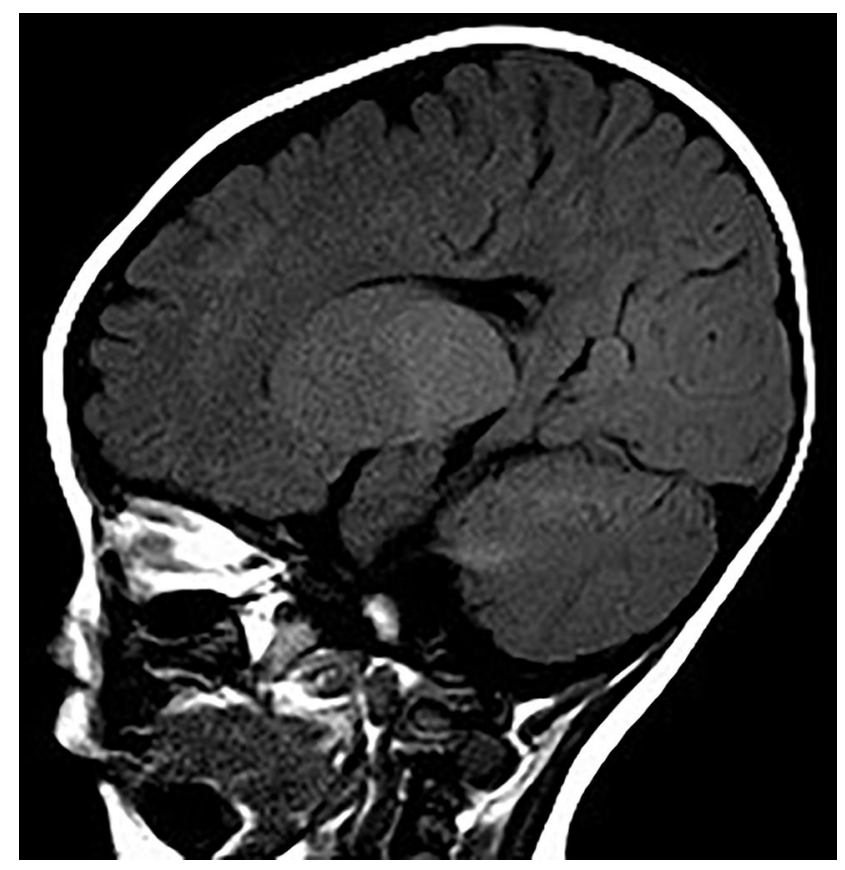

Figure 6 Sagittal MRI images at age of 9 months showing displayed diffusely and symmetrically abnormal signal in the white matter in bilateral cerebral hemispheres, cerebellar. 
and absence of myelination. All of the above features tended to suggest the diagnosis of PMD; however, whole-exome sequencing did not provide the genetic basis for PMD; thus, then we established PelizaeusMerzbacher-like disease (PMLD) as the patient's primary diagnosis.

\section{Genetic Results}

Whole-exome sequencing in our patient revealed compound heterozygous mutations in NCP1 (p. G911S, c2731G $>$ A and p.D128H, c382G $>$ C). c2731G $>$ A in exon 18 (chr18: 21119839) caused a point mutation that resulted in replacement of glycine with serine at position 911 (p. G911S). Point mutation of $\mathrm{c} 382 \mathrm{G}>\mathrm{C}$ in exon 4 (chcr18: 21148868) caused replacement of aspartic acid with histidine at position 128. The variants have not been reported according to the HGMDpro database. Moreover, we found no mutations in hypomyelinating leukodystrophyassociated genes, such as PLP1, GJC2, TUBB4A, GLB1, HEXA, HEXB, ERCC2, POLR3A, or RARS. ${ }^{8}$ According to the family's genetic screening, the patient's father had a mutation in NPC1 - c2731G $>$ A in exon 18 (chr18: 21119839), and the patient's mother also had a mutation in NPC1 - c382G $>$ C in exon 4 (chr18: 21148868) [Figures 7 and 8]. Thus, the complex heterozygous mutations in our patient were inherited from his father and mother, complying with Mendel's law of inheritance.

\section{Discussion}

In our report, we found compound heterozygous mutations in NCP1 (p. G911S, c2731G>A and p. D128H, c382G>C), which are thought to be associated with PelizaeusMerzbacher-like disease (PMLD).

As previously reported, variants in $\mathrm{NCP} 1$ are thought to be related to Niemann-Pick $\mathrm{C}$ disease. The dominant biochemical feature of Niemann-Pick type $\mathrm{C}$ is abnormal accumulation of low-density lipoprotein (LDL), which produces unesterified cholesterol in endosomes and lysosomes. ${ }^{9}$ The NPC1 (MIM 607623; chr 18q11-q12) gene encodes a membrane glycoprotein of 1278 amino acids, including an N-terminal domain (NTD), a middle luminal domain (MLD), a C-terminal domain (CTD), and 13 transmembrane helices (TMs). ${ }^{10}$ Moreover, studies on abnormal NPC1 protein have demonstrated that this protein is indeed a necessary part of intracellular cholesterol trafficking. ${ }^{9}$ To date, almost 490 mutations in the NPC1 gene have been reported, causing Niemann-Pick disease type $\mathrm{C}$. The clinical symptoms consist of abnormal visceral function (liver, spleen, or lung) and neurologic manifestations. ${ }^{7}$ However, the phenotype of our patient who showed mutations in the NPC1 gene was consistent with Pelizaeus-Melizaeus disease (PMD) rather than Niemann-Pick disease type C. The symptoms of our patient included nystagmus, dysmyotonia, ataxia, progressive motor dysfunction, mental retardation, and diffuse leukodystrophy on magnetic resonance imaging (MRI). All of
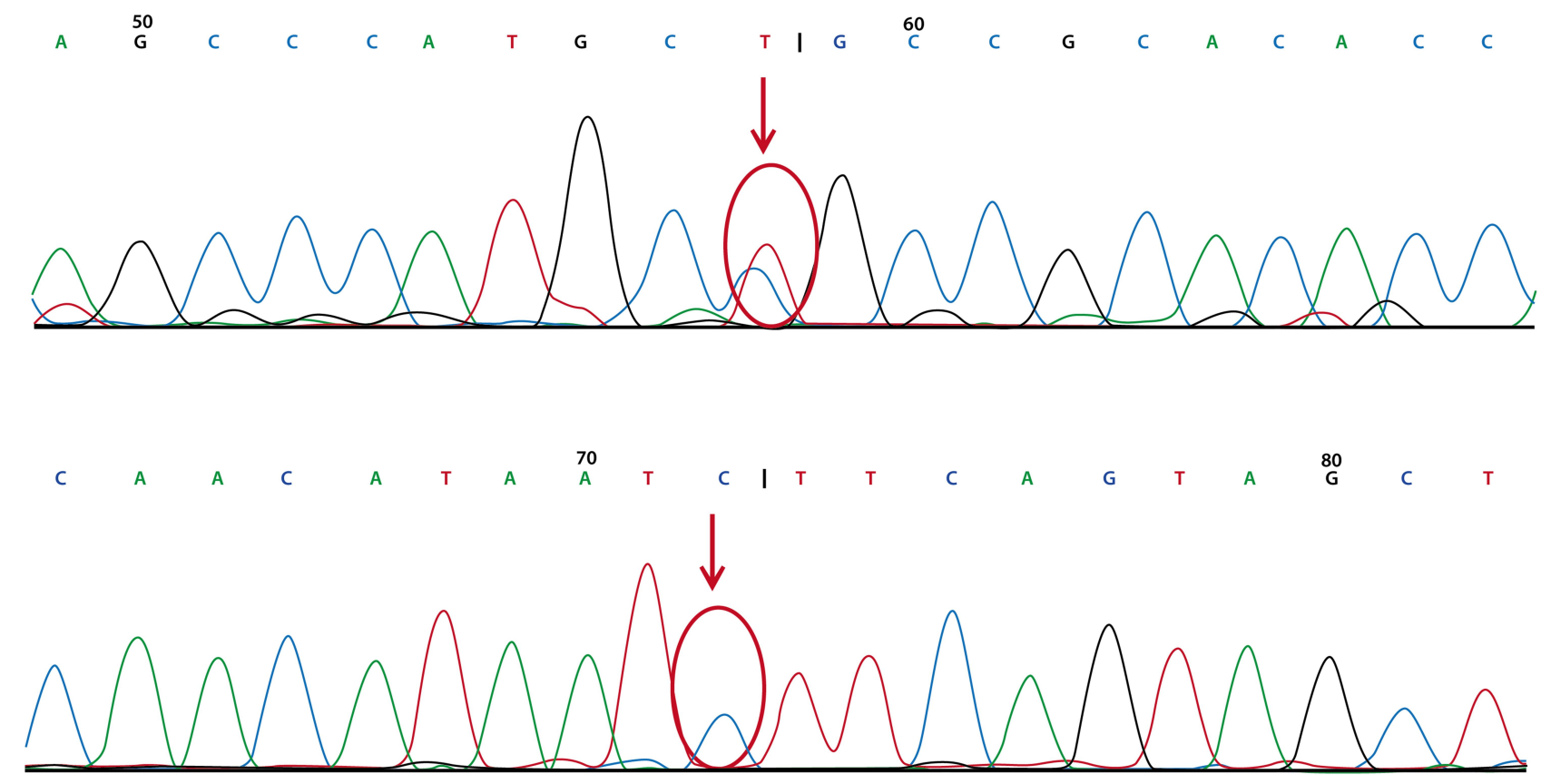

Figure 7 The patient's father had genetic mutation in chr18: 21 II9839 (upper red arrow) but no mutation in chr 18: 21 1488868 (under red arrow). 


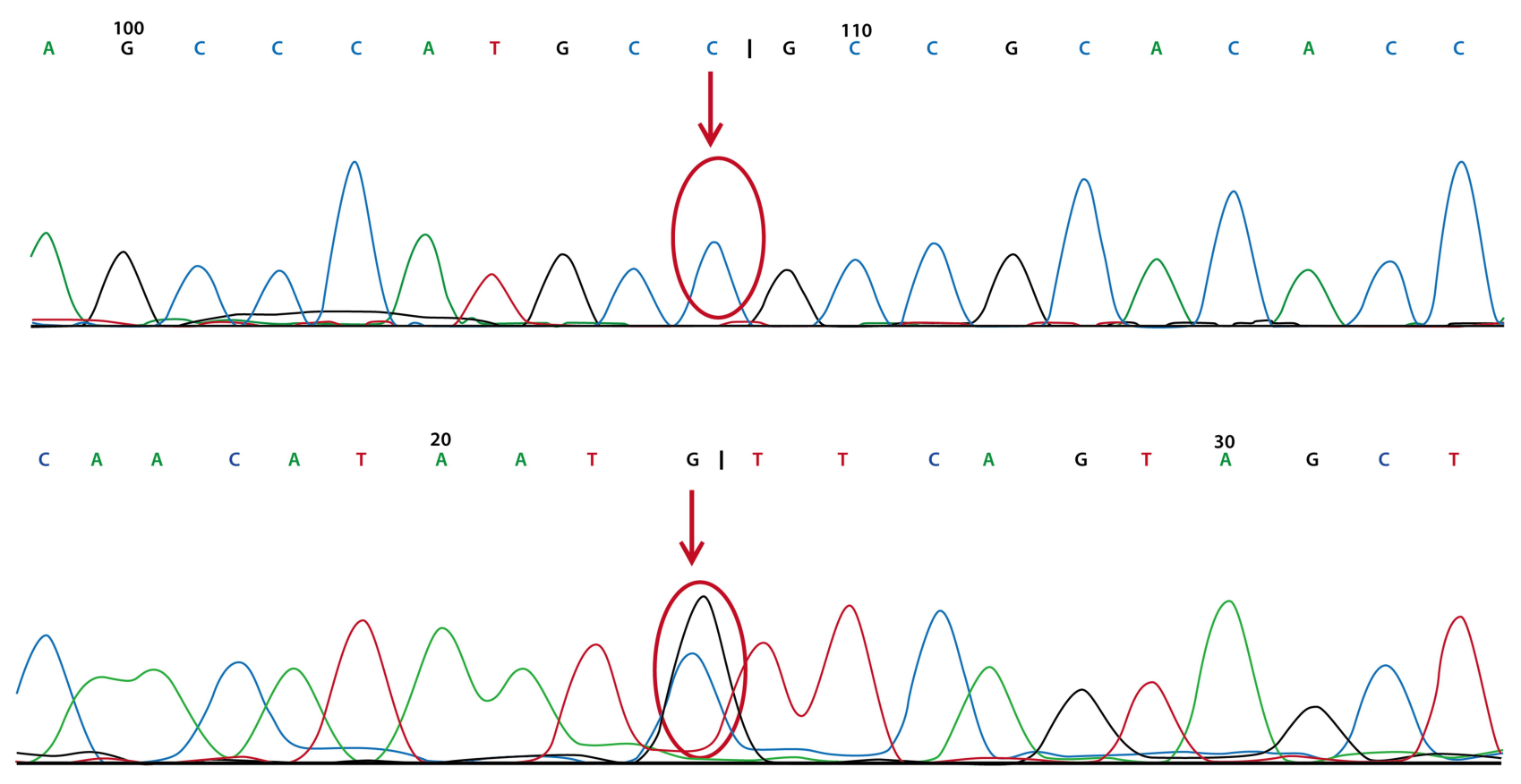

Figure 8 The patient's mother had genetic mutation in chr18: 211488868 (under red arrow) but no mutation in chr18: 21 II9839 (upper red arrow).

the clinical signs and imaging manifestations strongly suggest that the diagnosis of our patient with mutations in the NPC1 gene is more likely to be PMLD, although these mutations used to be related to Niemann-Pick disease type $\mathrm{C}$.

The NPC1 protein is an essential component of intracellular cholesterol trafficking and is localized in endosomes and lysosomes. ${ }^{9}$

The NPC1 protein is a membrane glycoprotein that consists of 13 transmembrane domains and three large loops localized to endosomes and lysosomes. These domains and loops play roles in regulating lipid metabolism when adequate levels of cholesterol are transmitted to the cell. ${ }^{9,10}$ Mutations in the NPC1 gene could result in abnormal lipid metabolism, causing large amounts of nonesterified cholesterol and glucoside sphingolipids to deposit in lysosomes. All these pathologic changes lead to a decrease in the self-renewal ability of neural stem cells and neurodegeneration. The myelin sheath is the membrane that surrounds the axon of a nerve cell and has a high lipid content - almost $70-75 \%$ of its dry weight. ${ }^{11}$ Therefore, abnormal lipid metabolism caused by mutations in the NPC1 gene could influence myelin formation, which was observed in our patient.

Llaci et al described a 12-year-old Caucasian/Hispanic male with pathogenic mutations in SNAP29 (p. Leu119AlafsX15, c.354DupG and p.0?, c.2T $>$ C), which have been associated with CEDNIK syndrome. ${ }^{12}$ Nafisinia et al reported two siblings with clinical symptoms of "PMD", while WES analysis confirmed a homozygous variant in the arginyl-tRNA synthetase (RARS) gene: NM_002887.3: c. [5A4G]; p.(Asp2Gly), which is possibly the cause of these symptoms. ${ }^{13}$

Numerous examples indicate that even the same exact mutation in the same zygosity can cause different diseases. ${ }^{14}$ Our patient with compound mutations in NPC1 could add a new example to the different diseases caused by the same gene.

\section{Concluding Remarks}

We found compound heterozygous mutations in the NPC1 gene in a patient with clinical symptoms of PMLD. Our findings expand the spectrum of NPC1-associated disorders and the gene spectrum of PMLD.

\section{Abbreviations}

PMLD, Pelizaeus-Merzbacher-like disease; PMD, PelizaeusMerzbacher disease; MRI, magnetic resonance imaging; WES, whole exome sequencing; NPC, Niemann-Pick disease type C; HLD1, hypomyelinating leukodystrophy-1; CNS, central nervous system; PLP1, proteolipid protein 1; GJC2, Gap junction protein gamma-2; Leu, leucine; Ala, alanine; Thr, threonine; NTD, N-terminal domain; MLD, middle luminal domain; CTD, C-terminal domain; CEDNIK, Cerebral 
Dysgenesis, Neuropathy, Ichthyosis and Keratoderma; RARS, arginyl-tRNA synthetase.

\section{Acknowledgments}

We thank the patient and his family for their informed consent for publication of this case.

\section{Author Contributions}

All authors made a significant contribution to the work reported, whether that is in the conception, study design, execution, acquisition of data, analysis and interpretation, or in all these areas; took part in drafting, revising or critically reviewing the article; gave final approval of the version to be published; have agreed on the journal to which the article has been submitted; and agree to be accountable for all aspects of the work.

\section{Funding}

This research is supported by the National Key R\&D Program of China (No. 2017YFC0211705) and Science \& Technology Department Program of Sichuan Province (No.2019YFS0144).

\section{Disclosure}

The authors have no conflicts of interest to disclose.

\section{References}

1. Lossos A, Elazar N, Lerer I, et al. Myelin-associated glycoprotein gene mutation causes Pelizaeus-Merzbacher disease-like disorder. Brain. 2015;138:2521-2536. doi:10.1093/brain/awv204
2. Inoue K. Pelizaeus-Merzbacher disease: molecular and cellular pathologies and associated phenotypes. Adv Exp Med Biol. 2019;1190:201-216.

3. Pouwels PJW, Vanderver A, Bernard G, et al. Hypomyelinating leukodystrophies: translational research progress and prospects. Ann Neurol. 2014;76:5-19. doi:10.1002/ana.24194

4. Lloyd-Evans E, Morgan A, Xingxuan H, et al. Niemann-Pick disease type $\mathrm{C} 1$ is a sphingosine storage disease that causes deregulation of lysosomal calcium. Nature Med. 2008;14:1247-1255. doi:10.1038/ nm.1876

5. Davies JP, Chen FW, Ioannou YA. Transmembrane molecular pump activity of Niemann-Pick C1 protein. Science. 2000;290:2295-2298. doi:10.1126/science.290.5500.2295

6. Vanier MT. Niemann-Pick disease type C. Orphanet J Rare Dis. 2010;5:16. doi:10.1186/1750-1172-5-16

7. Zervas M, Somers KL, Thrall MA, et al. Critical role for glycosphingolipids in Niemann-Pick disease type C. Current Biol. 2001;11:1283-1287. doi:10.1016/S0960-9822(01)00396-7

8. Ji H, Li D, Wu Y, et al. Hypomyelinating disorder in China: the clinical and genetic heterogeneity in 199 patients. PLoS One. 2018;13 (2):e0188869. doi:10.1371/journal.pone.0188869

9. Scott C, Ioannou YA. The NPC1 protein: structure implies function. Biochim Biophys Acta. 2004;1685:8-13. doi:10.1016/j. bbalip.2004.08.006

10. Andrea D, Stefania Z, Cinzia G, et al. Molecular genetics of Niemann-Pick type $\mathrm{C}$ disease in Italy: an update on 105 patients and description of 18 NPC1 novel variants. J Clin Med. 2020;9:679. doi: $10.3390 / \mathrm{jcm} 9030679$

11. Nave K-A, Werner HB. Myelination of the nervous system: mechanisms and functions. Annu Rev Cell Dev Biol. 2014;30:503-533. doi:10.1146/annurev-cellbio-100913-013101

12. Llaci L, Ramsey K, Belnap N, et al. Compound heterozygous mutations in Snap29 is associated with Pelizaeus-Merzbacher-like disorder (PMLD). Hum Genet. 2019. doi:10.1007/s00439-019-02077-7

13. Nafisinia M, Sobreira N, Riley L, et al. Mutations in RARS cause a hypomyelination disorder akin to Pelizaeus-Merzbacher disease. Eur J Human Gene. 2017;26:1-8. doi:10.1038/s41431-017-0024-z

14. Zhu X, Need AC, Petrovski S, Goldstein DB. One gene, many neuropsychiatric disorders: lessons from Mendelian disease. Nat Neurosci. 2014;17(6):773-781. doi:10.1038/nn.3713.
International Journal of General Medicine

\section{Publish your work in this journal}

The International Journal of General Medicine is an international, peer-reviewed open-access journal that focuses on general and internal medicine, pathogenesis, epidemiology, diagnosis, monitoring and treatment protocols. The journal is characterized by the rapid reporting of reviews, original research and clinical studies across all disease areas. The manuscript management system is completely online and includes a very quick and fair peer-review system, which is all easy to use. Visit http://www.dovepress.com/ testimonials.php to read real quotes from published authors. 